Diese Reductionen können angewandt werden:

Red. 1835 in den Jahren $1834-1839$

$\curvearrowright 1845>>1845-1847$

$\checkmark 1850$ D $\quad 1848-1854$

$\gg 1860>\quad \geqslant \quad 1855-1870$

$\checkmark 1875 \gg \times 1871-1879$

$\triangle 1883 .>1880-1883$

Die Jahre $1840-1844$ fehlen in dieser Reihe; es haben bei Aufstellung der Ephemeriden für diesen Zeitraum fortwährende theilweise Veränderungen der bis 1839 benutzten

Berlin, October 1893 .
Grundlagen stattgefunden, mit der Wirkung, dass dieselben im ganzen dem 1845 eingeführten System allmählich mehr und mehr angenähert, im einzelnen jedoch für diesen Zeitraum sehr heterogen; namentlich in Bezug auf $f(\delta)$ geworden sind.

Die Reductionstafel für $\mathrm{r}_{883}$ ist die bereits im Anhang zum B. J. 1884 mitgetheilte. In den Jahrgängen 1884-1893 führt der N. A. die Oerter des Nine year Catalogue, die Declinationen corrigirt nach der Tafel p. 27 der Einleitung zu diesem Catalog; im Jahrgang 1894 werden die Oerter des Ten year Catalogue eingeftihrt.

\title{
Ueber eine frühere Entdeckung des Cometen 1893 II (Rordame-Quénisset).
}

Nach ,Astronomy and Astrophysicse Nr. 118 p. 757 ist der Comet 1893 II bereits 1893 Juni I9 von Herrn $W . E$.Sperra in Randolph, Ohio entdeckt worden. Die genäherte Position des Cometen war:

$$
1893 \text { Juni } 19 \text { I }^{\mathrm{h}} \text { Ortszeit }=\text { Juni } 1920^{\mathrm{h}} 25^{\mathrm{m}} \text { M. Z. Gr. } a=2^{\mathrm{h}} 43^{\mathrm{m}} \delta=+17^{\circ} 30^{\circ}
$$
in diameter.

-It was of about the sixth magnitude or less, round, nebulous with condensation toward centre, perhaps $3^{\prime}$

Herr Sperra hat den Cometen an 13 Abenden bis Juli 10 verfolgt, ist aber bei dem Mangel an literarischen Hülfsmitteln in dem Glauben gewesen, dass er den Finlay'schen Cometen beobachtet habe, bis er erst durch die AugustNummer von , Astronomy and Astrophysics von seinem Irrthum überzeugt wurde.

Kiel 1893 Oct. 18 .

H. Kreutz.

\section{Zur Eigenbewegung des Sterns BD. $+\mathbf{2 5}{ }^{\circ} 2874$.}

(Siehe A. N. 2935 und 3054).

Meine mikrometrischen Verbindungen des Sterns BD. $+25^{\circ 2874}$, Gr. 9.5, mit dem Stern 5. Gr. Bradley r 924 (45 Bootis) ergaben:

\begin{tabular}{|c|c|c|c|c|c|c|c|c|c|c|c|c|}
\hline \multirow{2}{*}{ Epoche } & \multirow{2}{*}{ Nächte } & \multirow{2}{*}{ P. W. } & \multirow{2}{*}{ D. } & \multirow{2}{*}{$\Delta \alpha$} & \multirow{2}{*}{$\Delta \bar{o}$} & \multicolumn{2}{|c|}{ Red. auf I 890.0} & \multicolumn{2}{|c|}{ E. B. von Br. 1924} & \multicolumn{2}{|c|}{1890.0 (reducirte Werthe) } & \multirow{2}{*}{ Gew. } \\
\hline & & & & & & $\Delta \alpha$ & $\Delta 8$ & $\Delta \alpha$ & $\Delta 8$ & $\Delta \alpha$ & $\Delta 8$ & \\
\hline 1887.49 & I & $49^{\circ} 46^{\prime}$ & 252.29 & $+14^{5} .206$ & $+2 ' 42.95$ & -0.005 & +0.04 & -0.029 & +0.48 & $+14^{5} 172$ & $+2^{\prime} 43^{\prime}$ & $\boldsymbol{X}$ \\
\hline I $889.5^{6}$ & 3 & $49 \quad 16.0$ & 251.463 & +14.054 & +244.09 & -0.002 & +0.01 & -0.005 & +0.08 & +14.047 & +244.18 & 3 \\
\hline 1891.48 & 3 & $\begin{array}{ll}48 & 39.3\end{array}$ & 250.883 & +13.891 & +245.73 & 0.000 & -0.01 & +0.017 & -0.28 & +13.908 & +245.44 & 3 \\
\hline $1893.5^{\circ}$ & 4 & $\begin{array}{ll}48 & 0.6\end{array}$ & $250.5^{85}$ & +13.735 & +247.64 & +0.003 & -0.04 & +0.041 & -0.67 & +13.779 & +246.93 & 4 \\
\hline
\end{tabular}

Behandelt man diese Werthe nach der Methode der mit folgender Darstellung: kleinsten Quadrate, so ergiebt sich für $\mathrm{BD},+25^{\circ} \cdot 2874$ die Eigenbewegung von:

oder im grössten Kreise

$$
-0.0666 \text { und }+0.03 \mathrm{r}
$$

\begin{tabular}{|c|c|}
\hline $\begin{array}{r}+14.005 \\
018\end{array}$ & $\begin{array}{r}+2.45 " \circ 5 \\
44.46\end{array}$ \\
\hline 007 & 44.51 \\
\hline 012 & 44.72 \\
\hline
\end{tabular}

$$
\text { I". 102 Pos.-Winkel } 304^{\circ} 57^{\prime}
$$

Für Br. 1924 wurde nach Auwers die Eigenbewegung zu +o.o1 6 und -o"Igr angenommen.

Dresden 1893 Sept. 3 .

B. von Engelhardt. 